\title{
Biomechanical evaluation of 3D printed polycaprolactone scaffold for extraction socket preservation
}

\section{LEE Sang-Hwa ${ }^{1}$, SHIM Jin-Hyung ${ }^{2}$, LEE Jeoung-Seok², PARK Keun-Ho²}

Dept. of Dentistry St. Paul's Hospital College of Medicine The Catholic University of Korea ${ }^{1}$

Dept of Mechanical Engineering Korea Polytechinic University²

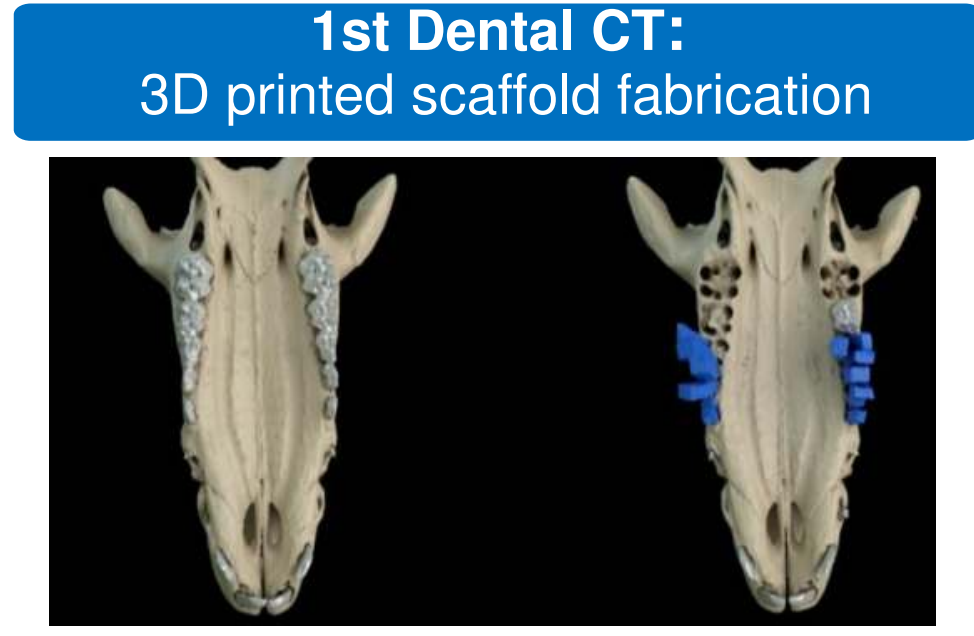

$2^{\text {nd }}$ Dental CT (image analysis):

Evaluation of accuracy of scaffold as space filler
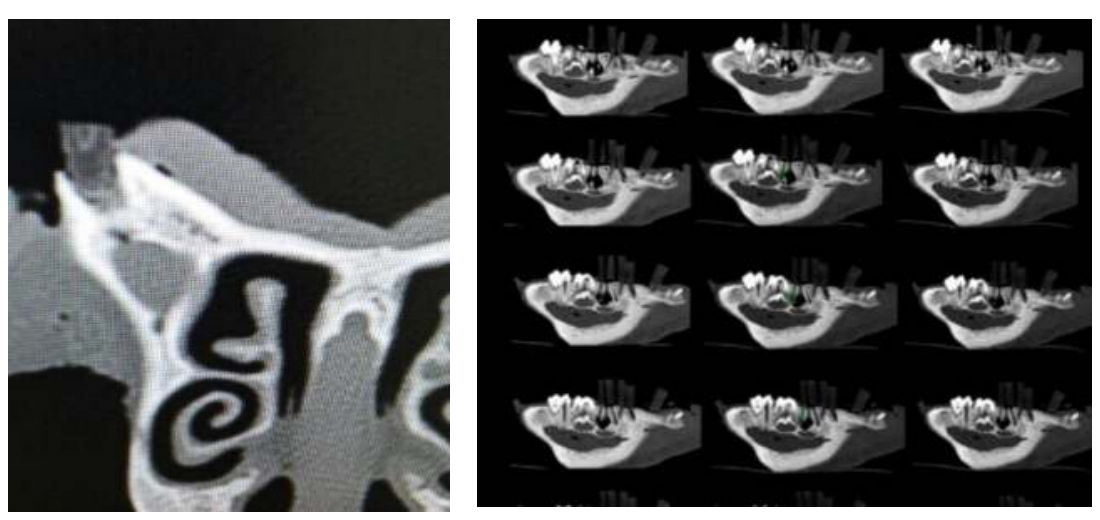

\section{Introduction}

\section{Polycaprolactone (PCL)}

It is the most utilized biomaterial in the 3D printed scaffold for the hard tissue regeneration including extraction socket preservation.

The mechanical properties of PC permit "interference fit" and it can be shaped by cutting with scalpel to fit on irregular shape of extraction socket

However, the efficacy and accuracy of PCL as space filler and biomechanical evaluation for ridge preservation is in debate.

\section{Purpose}

This paper is to evaluate 3D printed PCL scaffolds in different method the precision of adaptation as space filler

the mechanical stability

\section{Materials and Methods}

\section{Animals:}

Forty three dental roots from jaw specimens of two pigs (two maxillas and two mandibles)

\section{Scaffold fabrication:}

Before the extraction, dental CT images of tooth were sent for fabrication of custom made 3D printed root form PCL scaffold

The scaffolds were randomly divided into 2 groups

Group 1: 21 scaffolds printed with 100\% of original size $\rightarrow$ inserted to the extraction socket by interference fit

Group 2: 22 scaffold printed with 105\% of original size $\rightarrow$ shaped with scalpel to fit snuggle to the socket

\section{Surgery}

After the extraction, the group 1 scaffolds were inserted to the extraction socket by interference fit without any modeling and the group 2 scaffolds were shaped with scalpel to fit snuggle to the socket.
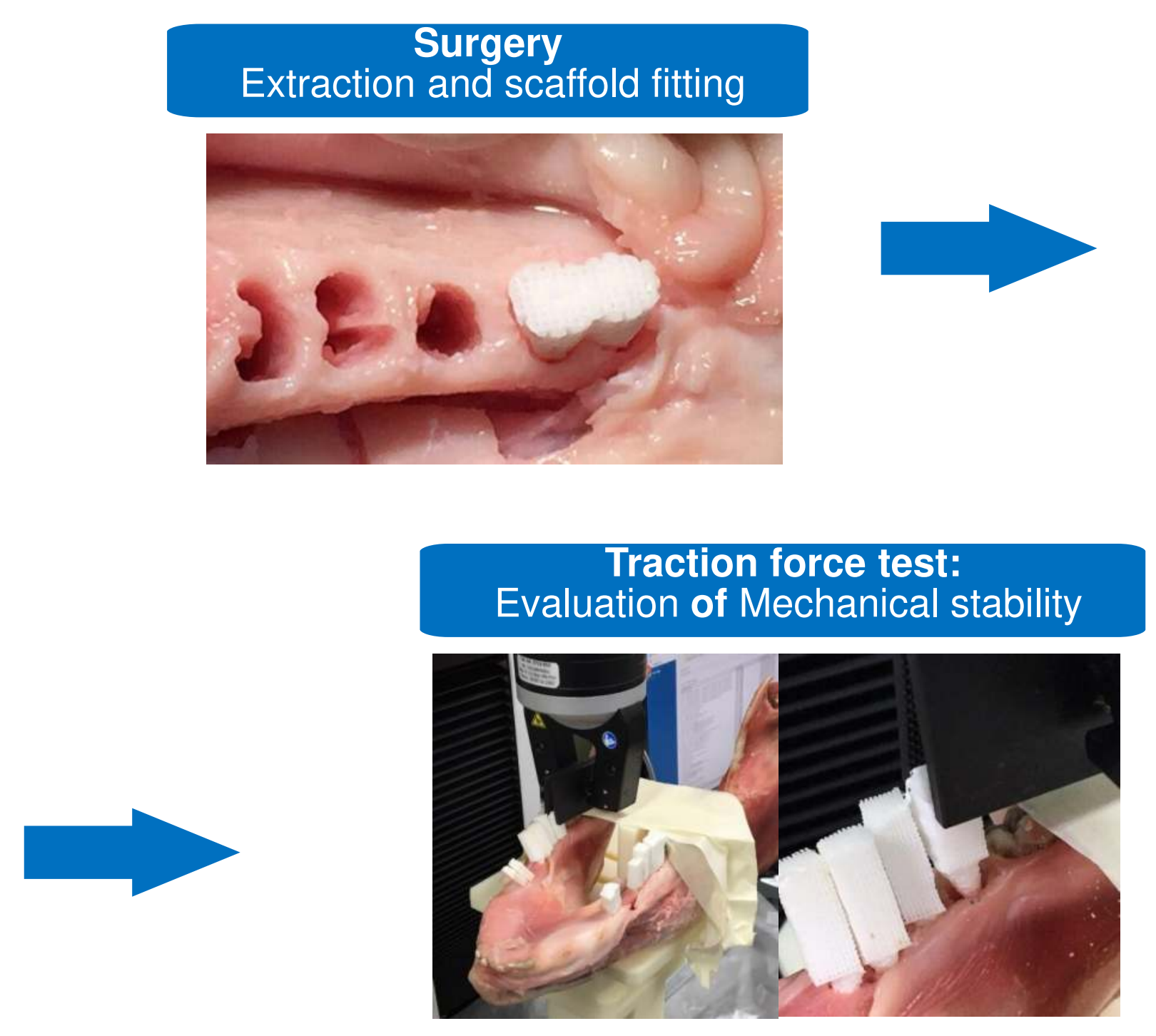

\section{Evaluation}

- Then second dental CT was taken to evaluate the accuracy of scaffolds as space filler using 3D image analysis.

- The mechanical stability of each scaffold was measured by traction force test.

- Statistical analysis: Wilcoxon rank sum test and Spearman rank correlation analysis

\section{Result}

\section{Precision of adaptation as space filler}

Fully filled extraction socket by the scaffold was no found.

The median percentage of volume filled by PCL in the extraction defect was $71.33 \%$ in group 1 and $73.64 \%$ in the group 2 .

There was no statistical differences between groups.

\section{Mechanical stability}

- The median traction force in group 1 was $20.71 \mathrm{~N}$ and group 2 was $27.34 \mathrm{~N}$.

There was no statistical differences between groups $(p=0.75)$.

\section{Conclusion}

This preliminary study shows no differences between two groups in mechanical stability and printing accuracy of scaffolds as space filler in the defect.

Clinical comfort and outcome should be evaluated for feasibility of 3D printed PCL scaffold

And further comparative studies in bio-mechanical properties and clinical outcomes with other composite 3D printed scaffold materials shall investigated for ridge preservation

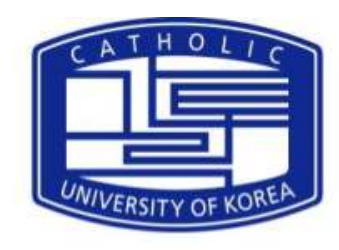

